$\$$ Research Square
Preprints are preliminary reports that have not undergone peer review.

They should not be considered conclusive, used to inform clinical practice, or referenced by the media as validated information.

\title{
Transsphenoidal Surgery of Invasive Corticotroph Adenomas: Results in A Series of 86 Consecutive Patients
}

\section{Congxin Dai}

Beijing Tongren Hospital

\section{Ming Feng}

PUMCH: Peking Union Medical College Hospital

Lin Lu

PUMCH: Peking Union Medical College Hospital

\section{Bowen Sun}

Beijing Tongren Hospital

\section{Yanghua Fan}

Beijing Tongren Hospital

\section{Xinjie Bao}

Beijing Tongren Hospital

\section{Yong Yao}

Beijing Tongren Hospital Department of Otorhinolaryngology and Head and Neck Surgery: Beijing

Tongren Hospital

\section{Kan Deng}

PUMCH: Peking Union Medical College Hospital

\section{Renzhi Wang}

Beijing Tongren Hospital

Jun Kang ( $\square$ kangjunty@126.com )

Beijing Tongren Hospital

\section{Research Article}

Keywords: invasive corticotroph adenomas, Transsphenoidal Surgery, Remission, Recurrence, Surgery, Immediate remission

Posted Date: August 18th, 2021

DOI: https://doi.org/10.21203/rs.3.rs-810471/v1 
License: (c) (i) This work is licensed under a Creative Commons Attribution 4.0 International License. Read Full License 


\section{Abstract}

Objective: Surgery is first-line treatment for corticotroph adenomas. Although most of corticotroph adenomas are noninvasive microadenomas that show expansive growth to surrounding tissues, a small subset of them is locally invasive and difficult to manage. The aim of this study was to evaluate surgical outcome of invasive corticotroph adenomas from a single-center.

Patients and Methods: The clinical features and outcomes of CD patients who underwent transsphenoidal surgery (TSS) between January 2000 and September 2019 at Peking Union Medical College Hospital were collected from medical records. The clinical, endocrinological, radiological, histopathological, surgical outcomes and a minimum 12-month follow-up of 86 consecutive CD patients with invasive corticotroph adenomas were retrospectively reviewed.

Results: Eighty-six patients with invasive corticotroph adenomas were included in the study. The average age at TSS was 37.7 years (range, 12 to 67 years), with a female-to-male ratio of 3.1:1 (65/21). The median duration of symptoms was 52.6 months (range, 1.0 to 264 months). The average of maximum diameter of tumor was $17.6 \mathrm{~mm}$ (range, 4.5-70 mm). All 86 patients with invasive corticotroph adenomas were performed TSS by microscopic or endoscopic approach. Gross-total resection was achieved in 63 patients (73.3\%), subtotal resection in $18(20.9 \%)$, and partial resection in $5(5.8 \%)$. After surgery, the overall postoperative immediate remission rate was $48.8 \%$ (42/86), $51.2 \%$ (44/86) of patients maintained persistent hypercortisolism. In 42 patients with initial remission, $16.7 \%(7 / 42)$ of them experienced a recurrence. In these patients with persistent disease and recurrent $C D$, data about further treatment was available for 30 patients. The radiotherapy was used for 15 patients, and 4 (26.7\%) of them achieved biochemical remission. Repeat TSS was performed in 5 patients, and none achieved remission. Medication was administrated in 4 patients, and one of them obtained disease control. Adrenalectomy was performed in 6 patients, and 5 (83.3 \%) achieved biochemical remission. At last follow-up, (33.3\%) 10 of 30 patients were in remission, and 20 patients still had persistent disease.

The remission rate in patients with invasive corticotroph adenomas who underwent gross-total resection and first TSS were significantly higher than that in patients undergoing subtotal resection, partial resection, and a second TSS (all P囚0.05). However, there was no significant difference in the remission rate between patient with different tumor size, Knosp Grade and surgical approaches (P凶0.05).

Conclusion: The management of invasive corticotroph adenomas remain a therapeutic challenge due to incomplete resection of invasive and/or a large adenoma. With application of multiple techniques assistance, approximately half of the patients could achieve gross-total resection and biochemical remission via TSS by experienced neurosurgeons. The extent of tumor resection and number of operations were associated with surgical remission rate in invasive corticotroph adenomas. If the remission was not achieved by surgery, other treatments including radiotherapy, medical therapy, and even bilateral adrenalectomy are required. 


\section{Introduction}

Cushing disease (CD) is defined as chronic hypercortisolemia, which is mainly caused by corticotroph adenomas. $C D$ is a relatively rare with an incidence of estimated at $0.7-2.4$ cases per million persons/year (1). If untreated or incompletely controlled, CD is associated with increased morbidity and higher mortality rate compared to general population (2). Transsphenoidal surgery (TSS) is considered the first-line treatment for patients with $C D$. However, the remission rate after TSS has been reported to vary widely from 59 to $94 \%$ (3). Most of corticotroph adenomas are non-invasive microadenomas with tumor diameter less than $8 \mathrm{~mm}(4,5)$. The surgical remission rates in these patients with noninvasive visible microadenomas frequently exceed greater than $80 \%$ (6). However, surgery for invasive corticotroph adenomas has represented challenge, and the remission rates in patients with extensive invasion into parasellar regions, such as the cavernous sinus, the skull base, or intracranially have tended to be relatively lower. Because invasive corticotroph adenomas are relatively rare, there is still no comprehensive study on large-scale cases of invasive corticotroph adenomas. To date, surgical outcome and perioperative complications of invasive corticotroph adenomas have not been clearly reported (7). The purpose of this study was to assess the surgical outcome and perioperative complications of patients with invasive corticotroph adenomas.

\section{Patients And Methods}

We conducted a retrospective analysis of consecutive patients with invasive corticotroph adenomas who underwent TSS at Peking Union Medical College Hospital (PUMCH) between January 2000 and September 2019. According to the guidelines of the PUMCH Medical Ethical Committee, clinical information on age, sex, clinical manifestation, preoperative biochemical parameters and imaging results, operative findings, postoperative hormone levels and pathology, long-term outcomes and follow-up were collected for analysis.

\section{Inclusion and exclusion criteria}

The inclusion criteria for this study were: 1) The CD was diagnosed based on the patient's history and results of a physical examination, laboratory tests, magnetic resonance imaging (MRI), and surgical findings; 2) a corticotroph adenomas was confirmed on immunohistopathological studies; 3 ) the invasion was recognized by intraoperatively findings; 4) with complete medical records and a minimum 12-month follow-up. According to the Knosp grade classification (8), if tumors were classified as Knosp grade of 1 or 2 based on preoperative MRI results, but the invasion was confirmed intraoperatively, these patients were also included into this study. If the tumors were classified as Knosp grade of 3 or 4 according to the preoperative MRI results, but no invasion was recognized intraoperatively, these patients were excluded from the study. Patients were excluded if the surgery was aborted because of carotid artery bleeding, or surgery was undergoing via transcranial approach, or the medical records were not complete, or lack of pathological report or follow-up data. 


\section{Diagnosis of Corticotroph Adenomas}

Most patients were diagnosed with invasive corticotroph adenomas by a pituitary multidisciplinary team (MDT) including members from the Departments of Neurosurgery, Endocrinology, Radiology and Pathology. As we described in previous study(9).All invasive corticotroph adenomas were diagnosed based on clinical manifestation, endocrine examinations, radiological examinations, intraoperative observation, and pathological results. In our center, all CD patients underwent comprehensive biochemical tests, including morning serum cortisol and ACTH, 24h-urine free cortisol (UFC), and a combined low-dose and high-dose dexamethasone suppression test (LDDST and HDDST). The methods of LDDST and HDDST has been described in detail in our previous study(10). If 24-h UFC and cortisol were not suppressed on the LDDST but was suppressed on the HDDST, CD was diagnosed. Based on our previous research results, the sensitivity of combined LDDST and HDDST to diagnose CD is $88.53 \%$ in our center(11).

Preoperatively, all patients underwent pituitary contrast-enhanced MRI and/or dynamic gadoliniumenhanced MRI. Images were independently reviewed by one neuroradiologist and at least one neurosurgeon, to evaluate the invasiveness of tumors, and ensure that agreement was achieved on Knosp grade which was descripted previously(8). Adenomas of Knosp Grade 0-2 were defined as noninvasive PAs, and adenomas of Knosp Grade 3-4 were defined as invasive PAs, respectively. According to the maximal diameter, adenomas were categorized as microadenoma $(<1 \mathrm{~cm})$, macroadenoma $(<4 \mathrm{~cm})$ and giant adenoma $(\geq 4 \mathrm{~cm})$.

\section{Surgical procedures}

All TSSs were performed in Department of Neurosurgery in PUMCH. Almost all the included patients were operated on by the same two experienced surgeons (Renzhi Wang and Ming Feng). The adenomas were removed by transsphenoidal microscopic, endoscopic approach or combination of them. The extended transsphenoidal approach assisted by multiple techniques was performed in some invasive macroadenomas or giant adenomas as previously described (12). In surgery, standard transsphenoidal PAs resection via microscopic approach or endoscopic approach was performed after broadly opening the sella floor. The tumor localized in the intrasellar and suprasellar region was removed first, then the residual tumor in the CS was inspected. The medial wall of the CS was explored under direct vision, soft tumor tissue was removed using suction and ring curettes. After the location of the ICA using intraoperative mini-Doppler. The anterior wall of the CS was opened carefully, then the soft tumors located lateral to the ICA was removed by suction and ring curettes. If the tumor was exposed insufficiently, the ICA could be gently pushed to increase visualization for further exposure. If the tumor was too hard to removed, or tumor adhesion to the ICA or nerves firmly, leave the tumor to avoid injury ICA or nerves. The residual tumors will be addressed by postoperative radiotherapy. Finally, the sella was reconstructed in all patients to avoid postoperatively cerebrospinal fluid (CSF) leakage and CNS infection.

\section{Classifications of intraoperative observed Invasion}


The cavernous sinus invasion was determined by the experienced neurosurgeons during surgery based on degree of invasion of the adenoma into the CS wall, the presence or absence of adenomas within the CS. After tumor removal, if the medial wall of CS was intact and smooth, absence of invasion was deemed. If the medial wall was not intact, intracavernous ligaments, ICA or nerve fibers were visible, the invasion was deemed.

\section{Extent of tumor resection}

The extent of tumor resection was evaluated through MRI examinations within 3 days and on 3 months after initial surgery. The gross total resection (GTR) was defined as no residual tumor was identified, neartotal resection. The subtotal resection (STR) was defined as more than $90 \%$ volume compared with the preoperative volume was removed, or when a definite residual tumor volume less than $10 \%$ compared with the preoperative volume was identified. Partial resection was defined as residual tumor volume greater than $10 \%(13)$.

\section{Histological analysis}

The resected specimens including pituitary adenomas were performed using routine $\mathrm{H}$ \& $\mathrm{E}$ histological stains and immunohistochemical tests for ACTH. The diagnosis of CD was confirmed if pathology under light microscopy demonstrated an adenoma with immunohistological staining positive for ACTH.

\section{Surgical outcome Measures}

After TSS, the endocrinological tests were performed within the first 7-day, 1 month, 3 months, and 12 months, respectively. As we described previously(14), immediate remission was defined as a serum cortisol level $<5 \mu \mathrm{g} / \mathrm{dL}$, and/or 24hUFC level fell below $20 \mu \mathrm{g}(56 \mathrm{nmol})$ within the first 7-day after TSS. Persistent disease was defined in patients as elevated postoperative cortisol levels and a need for additional therapy. Recurrence was defined in patients who achieved initial remission, followed by a rise in serum cortisol or 24hUFC and associated with clinical symptoms of CD. To assess the degree of tumor resection, postoperative MRI was done within 3 days and on 3 months after the surgery.

\section{Statistical Analysis}

Statistical significance for continuous variables was determined using the Student $t$ test or analysis of variance. Categorical variables were analyzed using either the Pearson $x^{2}$ test or Fisher exact test. Pearson $x^{2}$ testing for determination of statistical significance was performed by using either a $2 \cdot 2$ contingency table or test for independence if greater than 2 outcomes were being analyzed. Fisher exact test was used if any expected values were less than or equal to 5 . Statistical significance was defined as a P value less than.05. All statistical analyses were completed by using SPSS software, version 22 (SPSS, Inc, Chicago, Illinois).

\section{Results}




\section{Demographic and clinical characteristics of included patients}

From January 2000 to September 2019, 1381 patients with CD had TSS at the PUMCH, the intraoperative observed invasive corticotroph adenomas accounted for $7.5 \%$ (104/1381) of overall patients. Of these, 86 invasive corticotroph adenomas patients with complete medical records, pathological confirmation and a minimum 12-month follow-up were included this study. The detailed information was listed in Table 1. There are 21 male patients and 65 female patients, and the median duration of symptoms was 52.6 months (range, 1.0 to 264 months). The average age at TSS was 37.7 years (range, 12 to 67 years), and the mean follow-up time was 38.0 months (range, 12.0-147 months). 
Table 1

Demographic and clinical characteristics of 86 patients with invasive corticotroph adenomas

\begin{tabular}{|c|c|}
\hline Characteristics & value \\
\hline Total & 86 \\
\hline \multicolumn{2}{|l|}{ Gender } \\
\hline Female (n, \%) & $65(75.6 \%)$ \\
\hline Male $(n, \%)$ & $21(24.4 \%)$ \\
\hline Mean age, years (range) & $37.7(12-67)$ \\
\hline \multicolumn{2}{|l|}{ Number of operations } \\
\hline First (n, \%) & $66(76.7 \%)$ \\
\hline Repeat (n, \%) & $20(23.3 \%)$ \\
\hline Disease duration, months (range) & $52.6(1-264)$ \\
\hline BMI & $26.6(18.9-36.7)$ \\
\hline Tumor size (mean) (range) & $17.6 \mathrm{~mm}(4.5-70.0 \mathrm{~mm})$ \\
\hline Microadenoma (n, \%), Diameter (mm) & $(28,32.6 \%) 6.9 \mathrm{~mm}$ \\
\hline Macroadenoma (n, \%), Diameter (mm) & $(54,62.8 \%) 20.0 \mathrm{~mm}$ \\
\hline Giant adenoma (n, \%), Diameter (mm) & $(4,4.7 \%) 60.6 \mathrm{~mm}$ \\
\hline Preoperative 24-h UFC level $(\mu \mathrm{g})$ & $721.4(71.3-3705.6)$ \\
\hline Preoperative serum cortisol level ( $\mu \mathrm{g} / \mathrm{dL})$ & $28.9(7.4-75.0)$ \\
\hline Preoperative ACTH level (ng/L) & $142.5(23.1-1250.0)$ \\
\hline \multicolumn{2}{|l|}{ Combined LDDST and HDDST test } \\
\hline Positive (n, \%) & $72(83.7 \%)$ \\
\hline Negative $(\mathrm{n}, \%)$ & $14(16.3)$ \\
\hline Follow-up time, months (range) & $38.0(12.0-147.0)$ \\
\hline
\end{tabular}

The median tumor diameter of overall patients was $17.6 \mathrm{~mm}$ (range $4.5-70.0 \mathrm{~mm}$ ). Of these, $32.6 \%$ $(28 / 86)$ patients demonstrated microadenomas with a median tumor diameter of $6.9 \mathrm{~mm}, 62.8 \%(54 / 86)$ patients demonstrated macroadenomas with a median tumor diameter of $20.0 \mathrm{~mm}$, and $4.7 \%(4 / 86)$ patients had giant adenomas with a median tumor diameter of $60.6 \mathrm{~mm}$, respectively. Mean preoperative body mass index (BMI) was 26.6 (range, $18.9-36.7 \mathrm{~kg} / \mathrm{m}^{2}$ ). The median preoperative 24 -h UFC levels, 
morning serum cortisol and ACTH were $721.4 \mu \mathrm{g}$ (range 71.3-3705.6), $28.9 \mu \mathrm{g} / \mathrm{dL}$ (range7.4-75.0) and $142.5 \mathrm{ng} / \mathrm{L}$ (range 23.1-1250.0), respectively. In these cases, 66 patients underwent first TSS, and 20 patients underwent repeated TSS, respectively. In 83.7\% (72/86) patients, LDDST was not suppressed but HDDST was suppressed in the combined LDDST and HDDST test.

\section{Prediction of intraoperative cavernous sinus invasion by preoperative MRI-based Knosp grade}

The radiological results represent the first essential step to evaluate invasiveness of PAs. However, the results of invasion evaluated using Knosp grade based on preoperative MRI are not always consistent with intraoperatively observed invasiveness. In this study, 1273 patients were identified as Knosp Grade 0, 1 and 2, and 108 patients were classified into Knosp Grade 3 and 4 respectively based on preoperative coronal MRI (Table 2). Based intraoperatively observed results, none of the Knosp Grade 0 and 1 based on preoperative coronal MRI had shown evidence of intraoperative invasion, and all the MRI-based Knosp Grade 4 patients demonstrated invasion intraoperatively. However, invasion was intraoperatively observed in 5.4\% (4 of 74) patients with MRI-based Knosp Grade 2, and no obvious invasion was found intraoperatively in $16.0 \%$ (8 of 50) patients with MRI-based Knosp Grade 3.

Table 2

Comparison of Results of Knosp grade based on preoperative MRI and surgically observed invasiveness

\begin{tabular}{|llcc|}
\hline Knosp grade based on preoperative MRI & \multicolumn{2}{l}{ Surgically observed invasiveness } & \multirow{2}{*}{ Total } \\
\cline { 2 - 3 } & Invasive & non-invasive & \\
\hline 0 and 1 & 0 & 1199 & 1199 \\
\hline 2 & 4 & 70 & 74 \\
\hline 3 & 42 & 8 & 50 \\
\hline 4 & 58 & 0 & 58 \\
\hline Total & 104 & 1277 & 1381 \\
\hline
\end{tabular}

\section{Surgical outcome of different subgroups}

Surgical outcomes of different subgroups based on different variables were shown in Table 3. Postoperatively, only $42 / 86(48.8 \%)$ of overall included patients achieved immediate remission after TSS, whereas 44/86 (51.2\%) patients still had persistent disease. Knosp grade was closely related to extent of tumor resection and biochemical remission. In this study, after TSS, only 55.6\% (20/36) patients with Knosp Grade 3 and 41.3\% (19/46) patients with Knosp grade 4 adenomas achieved biochemical remission respectively. However, 75\% (3/4) patients with Knosp Grade 2 adenomas achieved remission, which were higher than that in the patients with Knosp grade 3 and 4 adenomas, but did not reach statistical significance (All P>0.05). 
Table 3

Rate of immediate remission and Recurrence by subgroups based on different variables

\begin{tabular}{|c|c|c|c|c|}
\hline Variable & Num. of patients & Remission & Persistence & $P$ value \\
\hline Total patients & 86 & $42 / 86(48.8 \%)$ & $44 / 86(51.2 \%)$ & \\
\hline \multicolumn{5}{|l|}{ Degree of invasion } \\
\hline Knosp Grade 2 (a) & $4(4.7 \%)$ & $3 / 4(75 \%)$ & $1 / 4(25 \%)$ & a vs $b: P=0.46$ \\
\hline Knosp Grade 3 (b) & $36(41.9 \%)$ & $20 / 36(55.6 \%)$ & $16 / 36(44.4 \%)$ & a vs c: $P=0.19$ \\
\hline Knosp Grade 4 (c) & $46(53.5 \%)$ & $19 / 46(41.3 \%)$ & $27 / 46(58.7 \%)$ & b vs c: $P=0.20$ \\
\hline \multicolumn{5}{|l|}{ Extent of tumor resection } \\
\hline GTR (d) & $63(73.3 \%)$ & $38 / 63(60.3 \%)$ & 25/63(39.7\%) & $d$ vs e: $P=0.004$ \\
\hline STR (e) & $18(20.9 \%)$ & $4 / 18(22.2 \%)$ & 14/18(77.8\%) & $d$ vs $f: P=0.008$ \\
\hline PR (f) & $5(5.8 \%)$ & $0 / 5(0 \%)$ & $5 / 5(100 \%)$ & e vs $f: P=0.25$ \\
\hline \multicolumn{5}{|l|}{ Tumor size } \\
\hline Microadenomas (g) & $28(32.6 \%)$ & $17 / 28(60.7 \%)$ & $11 / 28(39.3 \%)$ & $\mathrm{g}$ vs $\mathrm{h}: \mathrm{P}=0.12$ \\
\hline Macroadenomas (h) & $54(62.8 \%)$ & $23 / 54(42.6 \%)$ & $31 / 54(57.4 \%)$ & g vs i: $P=0.18$ \\
\hline Giant adenomas (i) & $4(4.7 \%)$ & $1 / 4(25.0 \%)$ & $3 / 4(75.0 \%)$ & h vs i: $P=0.49$ \\
\hline \multicolumn{5}{|l|}{ Approach } \\
\hline Microscopic approach (j) & $31(36.0 \%)$ & $14 / 31(45.2 \%)$ & $17 / 31(54.8 \%)$ & \\
\hline Endoscopic approach (k) & $55(64.0 \%)$ & $28 / 55(50.9 \%)$ & $27 / 55(49.1 \%)$ & j vs k: $P=0.61$ \\
\hline \multicolumn{5}{|l|}{ Number of operations } \\
\hline First TSS (I) & $66(76.7 \%)$ & $38 / 66(57.6 \%)$ & $28 / 66(42.4 \%)$ & \\
\hline Repeat TSS (m) & $20(23.3 \%)$ & $4 / 20(20.0 \%)$ & $16 / 20(80.0 \%)$ & I vs $\mathrm{m}: \mathrm{P}=0.003$ \\
\hline
\end{tabular}

Extent of tumor resection has been considered one of the most important factors affecting the surgical outcomes. In this study, GTR was achieved in 73.3\% (63/86) patients, and remission was obtained in $60.3 \%$ (38/63) of these patients. STR was performed in $20.9 \%$ (18/86) patients, and $22.2 \%(4 / 18)$ of them achieved remission. PR were performed in $5(5.8 \%)$ patients, and none achieved initial remission. The remission rate in patient performed with GTR was significantly higher than that in the patients with STR and RR( all $P<0.05)$.

Tumor size maybe another important factor correlated with outcomes of surgery. In this study, total 86 included patients had 28(32.6\%) microadenomas, 54(62.8\%) macroadenomas and 4(4.7\%) giant 
adenomas, and the postoperative remission rate in these patients were $60.7 \%(17 / 28), 42.6 \%(23 / 54)$ and $25.0 \%(1 / 4)$, respectively. However, there is no significant deference in remission rate among three groups ( all $P>0.05)$.

TSS can be performed by microscopic or endoscopic approach, whether endoscopic approach yielded higher remission rate over the microscopic approach is still controversial. In this study, there were 31 patients underwent microscopic approach and 55 patients underwent endoscopic approach respectively. However, no significant difference in the remission rate was observed between the two techniques in patients with invasive corticotroph adenomas $(P=0.61)$.

To further analyze the effect of the number of operations on the surgical outcome of invasive corticotroph adenomas, patients were subclassified into two groups based on the status of first or repeated TSS. In this study, 66 patients underwent first TSS and 20 patients underwent repeated TSS respectively. In the patients who underwent repeated TSS, only $20.0 \%(4 / 20)$ of them achieved immediate remission, which is significantly lower than the remission rate $(57.6 \%, 38 / 66)$ in the patients who underwent first TSS ( $p=0.003)$.

\section{Perioperative complications}

The surgery for invasive corticotroph adenomas usually was related to the high incidence of complications. In this series, the most common complication was intraoperatively CSF leakage, which occurred in in $24.4 \%$ (21/86) patients (Table 4). The sellar reconstruction by packing of the sphenoid sinus and sella turcica with fat, muscle and nasoseptal flap for all 21 patients with intraoperatively CSF leakage. However, there still were 5 patients having postoperative CSF leakage. Of these, 3 patients were successfully treated by repeated sellar reconstruction and external lumbar drain. Two patient who experienced CSF leakage and CNS infection was treated with antibiotics. After TSS, 15.1\% (13/86) patients experienced transient diabetes insipidus. Of these, 9 of 13 recovered after medical therapy, and 4 patients developed permanent diabetes insipidus and needed lifelong treatment with Desmopressin Acetate. After surgery, 5.8\% (5/86) patients experienced hypopituitarism and received hormone replacement therapy. Five patients (5.8\%) experienced partial abducent nerve palsy and three patients (3.5\%) experienced partial oculomotor nerve palsy respectively, and all recovered completely within 1 year. Three patients showed visual deterioration after TSS, and two of them experienced various degrees of improvement of visual dysfunction. One patient who was undergoing repeated TSS experienced ICA rupture, the rupture was controlled by direct compression, and underwent digital subtraction angiography immediately. The ICA rupture was blocked by the stent, and the patient recovered finally without neurological deficits. 
Table 4

Perioperative complications of CD patients based on Knosp Grade

\begin{tabular}{|llllll|}
\hline Results & Overall & $\begin{array}{l}\text { Knosp } \\
\text { Grade 2 }\end{array}$ & $\begin{array}{l}\text { Knosp } \\
\text { Grade 3 }\end{array}$ & $\begin{array}{l}\text { Knosp } \\
\text { Grade 4 }\end{array}$ & $\begin{array}{l}\text { P } \\
\text { value }\end{array}$ \\
\hline Intraoperatively CSF leakage & $\begin{array}{l}21 / 86 \\
(24.4 \%)\end{array}$ & $0 / 4(0 \%)$ & $\begin{array}{l}8 / 36 \\
(22.2 \%)\end{array}$ & $\begin{array}{l}13 / 46 \\
(28.3 \%)\end{array}$ & $P>0.05$ \\
\hline $\begin{array}{l}\text { Postoperatively CSF leakage } \\
(\%)\end{array}$ & $5 / 86(5.8 \%)$ & $0 / 4(0 \%)$ & $1 / 36(2.8 \%)$ & $4 / 46(8.7 \%)$ & $P>0.05$ \\
\hline Transient diabetes insipidus & 13 & $1 / 4(25.0 \%)$ & $\begin{array}{l}4 / 36 \\
(11.1 \%)\end{array}$ & $\begin{array}{l}8 / 46 \\
(17.4 \%)\end{array}$ & $P>0.05$ \\
\hline $\begin{array}{l}\text { Permanent diabetes } \\
\text { insipidus }\end{array}$ & $4 / 86(15.1 \%)$ & & $2 / 36(5.6 \%)$ & $2 / 46(4.3 \%)$ & $P>0.05$ \\
\hline $\begin{array}{l}\text { Postoperative } \\
\text { hypopituitarism }\end{array}$ & $5 / 86(5.8 \%)$ & $1 / 4(25.0 \%)$ & $2 / 36(5.6 \%)$ & $2 / 46(4.3 \%)$ & $P>0.05$ \\
\hline $\begin{array}{l}\text { Partial abducent nerve palsy } \\
\text { (\%) }\end{array}$ & $5 / 86(5.8 \%)$ & $0 / 4(0 \%)$ & $2 / 36(5.6 \%)$ & $3 / 46(6.5 \%)$ & $P>0.05$ \\
\hline $\begin{array}{l}\text { Partial oculomotor nerve } \\
\text { palsy (\%) }\end{array}$ & $3 / 86(3.5 \%)$ & $0 / 4(0 \%)$ & $2 / 36(5.6 \%)$ & $1 / 46(2.2 \%)$ & $P>0.05$ \\
\hline Impaired vision & $3 / 86(3.5 \%)$ & $0 / 4(0 \%)$ & $1 / 36(2.8 \%)$ & $2 / 46(4.3 \%)$ & $P>0.05$ \\
\hline CNS infection (\%) & $2 / 86(2.3 \%)$ & $0 / 4(0 \%)$ & $1 / 36(2.8 \%)$ & $1 / 46(2.2 \%)$ & $P>0.05$ \\
\hline \begin{tabular}{llll} 
ICA rupture (\%) \\
\hline
\end{tabular} & $1 / 86(1.2 \%)$ & $0 / 4(0 \%)$ & $0 / 36(0 \%)$ & $1 / 46(2.2 \%)$ & $P>0.05$ \\
\hline
\end{tabular}

\section{Recurrence}

Recurrence was observed in $16.7 \%$ (7/44) all patients with initial remission, and the median time to recurrence for these patients was 33.0 months (range 2.0-105.0months) (Table 5). However, no patients with Knosp Grade 2 was found to be recurrence. No significant difference in recurrence rate was observed between the patients with Knosp Grade $3(15.0 \%, 3 / 20)$ and Knosp Grade 4 adenomas $(21.1 \%, 4 / 19)$ $(P>0.05)$.

Table 5

Recurrence outcome of CD patients with initial remission based on Knosp Grade.

\begin{tabular}{|llllll|}
\hline MRI Results & Overall & $\begin{array}{l}\text { Knosp } \\
\text { Grade 2 }\end{array}$ & $\begin{array}{l}\text { Knosp } \\
\text { Grade 3 }\end{array}$ & $\begin{array}{l}\text { Knosp Grade } \\
\mathbf{4}\end{array}$ & $\begin{array}{l}\text { P } \\
\text { value }\end{array}$ \\
\hline Number of Patients & $7 / 42(16.7 \%)$ & $0 / 3(0 \%)$ & $\begin{array}{l}3 / 20 \\
(15.0 \%)\end{array}$ & $4 / 19(21.1 \%)$ & $\begin{array}{l}\mathrm{P}= \\
0.62\end{array}$ \\
\hline $\begin{array}{l}\text { Median time to recurrence } \\
\text { (Ms) }\end{array}$ & $\begin{array}{l}33.0(2.00- \\
105.0)\end{array}$ & 0 & $\begin{array}{l}29.0(6.0- \\
56.0)\end{array}$ & $\begin{array}{l}36.0(2.0- \\
105.0)\end{array}$ & $P>0.05$ \\
\hline
\end{tabular}




\section{Further treatments for persistent and recurrent CD}

For those patients with persistent disease (44) and recurrent CD (7), data about further treatment were available for only 30 of 51 patients. Radiotherapy was used for 15 patients, and 4 (26.7\%) of them achieved biochemical remission. Repeat TSS was performed in 5 patients, but none achieved remission. Medication was administered in 4 patients, and only one obtained disease control. Adrenalectomy was performed in 6 patients, and 5 (83.3\%) achieved biochemical remission. At the last follow-up, $33.3 \%$ (10 of 30 ) patients were in remission, and 20 patients still had persistent disease (Table 6).

Table 6

Further treatments and Remission Rate in persistent and recurrent patients with invasive corticotroph adenomas

\begin{tabular}{|lllll|}
\hline Total /Remission Rate & Radiotherapy & Repeat TSS & Medical therapy & Adrenalectomy \\
\hline Total $(10 / 30,33.3 \%)$ & $4 / 15(26.7 \%)$ & $0 / 5(0 \%)$ & $1 / 4(25.0 \%)$ & $5 / 6(83.3 \%)$ \\
\hline
\end{tabular}

\section{Discussion}

This study reports on the outcomes of TSS in 86 patients with invasive corticotroph adenomas who underwent at the PUMCH between 2000 and 2019. To our knowledge, this is one of the largest series on invasive corticotroph adenomas published till now. This study demonstrated that the immediate remission of the surgical approach and the long-term outcome after adjuvant therapies for patients with invasive corticotroph adenomas are unsatisfactory. Additionally, invasion evaluated using Knosp grade based on preoperative MRI not always accurately predict intraoperatively observed invasiveness.

Moreover, extent of tumor resection and number of operations significantly affected the surgical outcome of TSS for invasive corticotroph adenomas. However, the Knosp grade, tumor size, and technical factor is not associated with the surgical outcome.

Preoperative MRI-based Knosp grade classifications play a crucial role in diagnosis of cavernous sinus invasion (CSI) of corticotroph adenomas. However, radiological results do not always discriminate between compression/extension and invasion of CS. In this study, only $84.0 \%$ (42 of 50) of the patients with MRI-based Knosp grade 3 was intraoperatively identified with CSI, and other 8 patients with Knosp grade 3 adenomas did not have CSI at surgery. Further, $5.4 \%$ (4 of 74) patients with MRI-based Knosp grade 2 adenomas were found invasion during surgery. These findings are consistent with previous studies $(15,16)$. Dickerman and colleagues (17) demonstrated that dural invasion was directly observed at surgery and was confirmed histologically in $62 \%$ of the patients with no adenomas were interpreted based on preoperative MRI. Lonser also reported that preoperative MRI accurately predicted dural invasion in only 4 patients (22\%) with CSI (18). Other previous studies also indicated that Knosp classification does not predict accurately the invasion of the Knosp grade 0-2 adenomas, thus their application in the prediction of invasion among microadenomas is limited (19). A recent Meta-Analysis also reported that the prevalence of CSI radiographically (43\%) was much higher than that (18\%) intraoperatively, and the radiologic criteria of Knosp 3-4 had the highest correlation with intraoperative 
CSI (20). Therefore, although MRI-based Knosp grade can reliably define the degrees of CSI in Knosp 3-4 larger tumors, it is often unreliable to define the absence of dural invasion in Knosp 0-2 microadenomas. To define more accurately invasion beyond the lateral tangential line between ICA segments respectively, Knosp updated the original grading system of invasion in PAs by establishing the subtypes of grade 3a and $3 \mathrm{~b}$ PAs in 2015 (15). However, up to $80 \%$ of CD patients present with a microadenoma, and there is no reliable grading system for microadenomas that predicts accurately CSI. Thus, more reliable grading systems of invasion for corticotroph adenomas are need.

For invasive corticotroph adenomas, complete surgical resection of tumor is difficult. Thus, lower remission rates of TSS have been reported in the patients with invasive corticotroph adenomas. However, $48.8 \%$ of patients with invasive corticotroph adenomas in our center achieved remission after TSS, which was higher than that reported in previous studies $(7,21)$. The main reasons for the higher remission rate may include the experienced neurosurgeon, intraoperative multiple techniques assistance and aggressive surgical procedure.

Surgery for invasive corticotroph adenomas has always been a challenge because of the highly complex anatomy of the CS and difficult in CS dissection. Thus, experienced neurosurgeon is essential to achieve complete tumor resection, biochemical remission, and avoid perioperative complications. In our center, almost all the surgery for CD patients were performed by Pro. Renzhi Wang and Ming Feng, who had experience with more than one thousand pituitary surgeries as previously described(22). Each year, more than one hundred of CD patients undergo pituitary surgery in our center, and a large part of them are invasive macroadenomas and recurrent $\mathrm{CD}(23)$. The number of surgical patients with $C D$ may be one of the largest centers. Therefore, large-scale surgical patients with $C D$ have accumulated rich surgical experience in management of patients with invasive corticotroph adenomas. Unfortunately, even in the hands of experienced surgeons, only about half of patients with invasive corticotroph adenomas could achieve remission after TSS. There are several studies also found that biochemical remission rate is related to the number of years of neurosurgical experience. Yap and colleagues reported that the first decade of neurosurgery experience was associated with lower remission rates than that with the second and third decade of neurosurgery experience(24). A recent meta-analysis also demonstrated that the possible association of neurosurgeons' experience with remission rates in CD patients (25). Therefore, neurosurgical experience may be one of main reasons for the higher remission rate of TSS in patients with invasive corticotroph adenomas.

Application of multiple techniques assistance maybe another important factor for higher remission rate of TSS in patients with invasive corticotroph adenomas. In this study, multiple techniques including neuronavigation and intraoperative Doppler ultrasonography were used intraoperatively for surgical assistance in most patients, which resulted in maximum tumor removal and a relatively low rate of perioperative complications. For larger and recurrent invasive corticotroph adenomas, neuronavigation and Doppler ultrasonography was used to determine the exact location of the ICA. These techniques can provide references for locating some important structures including the ICA, brainstem, and optic canal in real time during surgery(26). Precisely location these structures can prevent to injuries them, thereby 
decrease the frequency of perioperative complications. T. J. Owen also reported that using the neuronavigation system for localization the rostral and caudal margins of the pituitary fossa during TSS may decrease morbidity and surgical time (27). Doppler ultrasonography also has been shown to determine the exact location of the ICA and whether it exist an aneurysm, thereby avoid to injury ICA the during surgery (28). Therefore, these multiple techniques assistance used in surgery facilitate tumor resection, the operation safe and decrease perioperative complications in patients with corticotroph adenomas.

In our center, aggressive procedure was used to pursue a maximum safe removal of tumor. During the surgery, in order to accurately assess the dural invasion and to remove the tumor maximally, it is critical to widely expose of the anterior and inferior sella dura, even the medial dural wall of the cavernous sinuses. Thus, endoscopic extended transsphenoidal surgery was used for most lager invasive corticotroph adenomas, which provide direct visualization for resection of the tumors invading the CS and suprasellar (28). Recently, the incision of the CS wall also has been performed for PAs invading the $\mathrm{CS}$, which resulted in a higher GTR rate(12). Therefore, rich surgical experience, wide exposure by endoscopic extended transsphenoidal surgery and incision of the CS, and intraoperative assistance of combined neuronavigation and intraoperative Doppler ultrasonography are the main reasons for the higher remission rate in our center.

Identification of the factors affecting surgical outcomes is very is important for predicting the prognosis of patients with invasive corticotroph adenomas. In this study, we found that the remission rate $(75 \%)$ in patients with Knosp grade 2 adenomas is higher than that in patients with Knosp grade 3 adenomas $(55.6 \%)$ and Knosp grade 4 adenomas (41.3\%), but it did not reach the statistical significance. This result indicated that the MRI-based Knosp grade classifications was not related to immediate remission in invasive corticotroph adenomas, which is consistent with the results of most previous studies. Similarly, Wagenmakers (6) reported that the remission was achieved in $50.0 \%$ of patients with Knosp grade 2 invasion, $37.5 \%$ of patients with Knosp grade 3 invasion, and $33.3 \%$ patients with Knosp grade 4 invasion, respectively. However, another study by Witek showed that the immediate postoperative remission depended on invasiveness based on Knosp grades 3 and 4 for macroadenomas (29). However, only 4 patients with Knosp grade 2 adenomas were included in the present study, and more large-scale studies are need to further verify this conclusion.

Whether the adenoma size affect the surgical remission in patients with corticotroph adenoma is remaining controversial. In our study, patients with invasive microadenomas had higher immediate remission rates than that in patients with invasive macroadenomas and invasive giant adenomas, however, it does not reach statistical significance. This is consistent with the results from some previous studies. Starke reported that there was no significant difference in remission among patients with microadenomas and macroadenomas(31). Feng also reported that CD patients with macroadenomas and microadenomas had similar remission rate after TSS(11). On the contrary, a few studies have opposite conclusions, Blevins(32) indicated that CS invasion and the presence of a tumor diameter $\geq 2.0$ $\mathrm{cm}$ were characteristics associated with an increased likelihood of residual disease after surgery. Other 
studies also demonstrated an inverse correlation between remission rates and tumor size in patients with CD $(33,34)$. However, most of previous studies reported that the effect of tumor diameter on remission rate in patients with $C D$, but not patients with invasive corticotroph adenoma. Therefore, more studies on potential factors predicting surgical outcomes in patients with invasive corticotroph adenomas are needed.

In this study, we found that the repeated TSS for invasive corticotroph adenomas has been shown significant lower remission rates compared with the first TSS. The main reasons for this result maybe that the destruction of the original anatomy and scar formation within the recurrent tumor. These factors make the surgery more difficult and dangerous. Valderrábano and colleagues (35) also demonstrated that the repeat TSS for CD is associated to a lower remission rate and a higher risk of recurrence, which is consistent to our results. However, the large-scale clinical study on results of repeat TSS for invasive corticotroph adenomas is limited, and further studies are needed.

Depending on neurosurgeon's preference, TSS for invasive corticotroph adenomas could be performed by microscopic or endoscopic approach. Compared with microscopic approach, the endoscopic approach provides a broader surgical view of pituitary region, including lateral edges of the sella and cavernous sinuses. However, in this study, no significant difference was found in the remission rates among the patients with invasive corticotroph adenomas who underwent microscopic TSS and endoscopic TSS. The microscopic and endoscopic techniques were used in combination for subset of patients with invasive corticotroph adenomas, which might explain why endoscopic versus microscopic technique yielded the similar remission rate in our center. This result in accordance with one recent meta-analysis, which indicated that comparisons of remission rates by endoscopic versus microscopic technique yielded the same results(36). However, another meta-analysis demonstrated that the endoscopic TSS reaches comparable results for microadenomas, and probably better results for macroadenomas than microscopic TSS for CD patients (37). To date, the data about comparisons of remission rates by endoscopic versus microscopic technique in invasive corticotroph adenomas is limited, more studies on comparison of the two techniques in patients with invasive corticotroph adenomas at the same institution are needed.

It is difficult to manage patients with persistent or recurrent invasive corticotroph adenomas. Therapy options include radiation therapy, repeated TSS, medical therapy, and as a final step bilateral adrenalectomy. These treatments have their own advantages and disadvantages, however, there is no consensus on which treatment is preferable. In our center, an individual-based comprehensive treatment was discussed by a multidisciplinary team (MDT) with collaborating experts. However, even if comprehensive treatments were used, the prognosis of these patients is still poor, and more effective treatment are need.

\section{Limitations}


Although this study is a larger patient cohort on the results of TSS on invasive corticotroph adenomas in a single institution, there are some limitations that deserve mention. First, because of the retrospective nature of the study, it is difficult to collect completely clinical information and long-term follow-up data for all patients. Some data on long-term follow-up and further treatments were missing, which may result in a bias of the results and conclusions on long-term outcomes and recurrence rate. Additional, information of survival or death for half of the included patients cannot be obtained, thereby the mortality results are not included and analyzed. Another limitation is that referral or selection bias must be taken into consideration because this was a single-center cohort study.

\section{Conclusions}

In summary, the remission rate of CD patients with invasive corticotroph adenomas was unsatisfactory due to incomplete resection of invasive and/or a large adenoma. Extent of tumor resection and number of operations were associated with surgical remission rate in invasive corticotroph adenomas. In contrast, Knosp grade, tumor size and surgical approach did not affect the remission rate of TSS. In addition, further treatment including radiotherapy, repeated operation, medical therapy, and even bilateral adrenalectomy are required for persistent or recurrent invasive corticotroph adenomas.

\section{Declarations}

\section{AUTHOR CONTRIBUTIONS}

All authors listed have made a substantial, direct, and intellectual contribution to the work, and approved it for publication.

\section{Declaration of interest}

None of the authors have potential financial conflicts of interest related to this article. The financial support for this study was provided by the Scientific Research Project of Capital Health Development in 2018 (grant number: 2018-4-4018), CAMS Innovation fund for Medical Science (grant number: CIFMS, 2017-12M-2-005) and Beijing Natural Science Foundation (grant number: 7182137). The funding institutions had no role in the design of the study, data collection and analysis, the decision to publish, or the preparation of the manuscript.

\section{References}

1. C. Steffensen, A. M. Bak, K. Zøylner Rubeck and J. O. L. Jørgensen: Epidemiology of Cushing's Syndrome. Neuroendocrinology, 92(1), 1-5 (2010) doi:10.1159/000314297

2. R. N. Clayton, D. Raskauskiene, R. C. Reulen and P. W. Jones: Mortality and Morbidity in Cushing's Disease over 50 Years in Stoke-on-Trent, UK: Audit and Meta-Analysis of Literature. The Journal of Clinical Endocrinology \& Metabolism, 96(3), 632-642 (2011) doi:10.1210/jc.2010-1942 
3. A. G. Ioachimescu: Prognostic Factors of Long-Term Remission After Surgical Treatment of Cushing's Disease. Endocrin. Metab. Clin., 47(2), 335-347 (2018) doi:10.1016/j.ecl.2018.02.002

4. P. C. Johnston, L. Kennedy, A. H. Hamrahian, Z. Sandouk, J. Bena, B. Hatipoglu and R. J. Weil: Surgical outcomes in patients with Cushing's disease: the Cleveland clinic experience. Pituitary, 20(4), 430-440 (2017) doi:10.1007/s11102-017-0802-1

5. M. Solak, I. Kraljevic, T. Dusek, A. Melada, M. M. Kavanagh, V. Peterkovic, D. Ozretic and D. Kastelan: Management of Cushing's disease: a single-center experience. Endocrine, 51(3), 517-523 (2016) doi:10.1007/s12020-015-0695-6

6. M. A. E. M. Wagenmakers, H. D. Boogaarts, S. H. P. P. Roerink, H. J. L. M. Timmers, N. M. M. L. Stikkelbroeck, J. W. A. Smit, E. J. van Lindert, R. T. Netea-Maier, J. A. Grotenhuis and A. R. M. M. Hermus: Endoscopic transsphenoidal pituitary surgery: a good and safe primary treatment option for Cushing's disease, even in case of macroadenomas or invasive adenomas. Eur. J. Endocrinol., 169(3), 329-337 (2013) doi:10.1530/EJE-13-0325

7. Z. Zhuang, X. Liu, X. Bao, B. Pan, K. Deng, Y. Yao, W. Lian, B. Xing, H. Zhu, L. Lu, R. Wang and M. Feng: Invasive ACTH-secreting pituitary macroadenoma in remission after transsphenoidal resection: A case report and literature review. Medicine, 97(46), e13148 (2018) doi:10.1097/MD.0000000000013148

8. E. Knosp, E. Steiner, K. Kitz and C. Matula: Pituitary adenomas with invasion of the cavernous sinus space: a magnetic resonance imaging classification compared with surgical findings. Neurosurgery, 33(4), 610-7; discussion 617-8 (1993) doi:10.1227/00006123-199310000-00008

9. C. Dai, Y. Fan, X. Liu, X. Bao, Y. Yao, R. Wang and M. Feng: Predictors of Immediate Remission after Surgery in Cushing's Disease Patients: a large retrospective study from a single center. Neuroendocrinology (2020) doi:10.1159/000509221

10. M. Feng, Z. Liu, X. Liu, X. Zhang, X. Bao, Y. Yao, K. Deng, B. Xing, W. Lian, H. Zhu, L. Lu and R. Wang: Tumour lateralization in Cushing's disease by inferior petrosal sinus sampling with desmopressin. Clin. Endocrinol., 88(2), 251-257 (2018) doi:10.1111/cen.13505

11. M. Feng, Z. Liu, X. Liu, X. Bao, Y. Yao, K. Deng, B. Xing, W. Lian, H. Zhu, L. Lu and R. Wang: Diagnosis and Outcomes of 341 Patients with Cushing's Disease Following Transsphenoid Surgery: A SingleCenter Experience. World Neurosurg., 109, e75-e80 (2018) doi:10.1016/j.wneu.2017.09.105

12. X. Bao, K. Deng, X. Liu, M. Feng, C. C. Chen, W. Lian, B. Xing, Y. Yao and R. Wang: Extended transsphenoidal approach for pituitary adenomas invading the cavernous sinus using multiple complementary techniques. Pituitary, 19(1), 1-10 (2016) doi:10.1007/s11102-015-0675-0

13. M. A. de Paiva Neto, A. Vandergrift, N. Fatemi, A. A. Gorgulho, A. A. DeSalles, P. Cohan, C. Wang, R. Swerdloff and D. F. Kelly: Endonasal transsphenoidal surgery and multimodality treatment for giant pituitary adenomas. Clin. Endocrinol., 72(4), 512-519 (2010) doi:10.1111/j.1365-2265.2009.03665.x

14. C. Dai, S. Liang, X. Liu, Y. Fan, X. Bao, Y. Yao, K. Deng, L. Lu, R. Wang and M. Feng: Outcomes of Transsphenoidal Surgery in Cushing Disease Patients with Negative Pituitary Magnetic Resonance 
Imaging Findings: A Single-Center Experience. Endocr. Pract., 26(11), 1320-1330 (2020) doi:10.4158/EP-2020-0177

15. A. S. G. Micko, A. Wöhrer, S. Wolfsberger and E. Knosp: Invasion of the cavernous sinus space in pituitary adenomas: endoscopic verification and its correlation with an MRI-based classification. J. Neurosurg., 122(4), 803-811 (2015) doi:10.3171/2014.12.JNS141083

16. P. Mastorakos, D. G. Taylor, C. Chen, T. Buell, J. H. Donahue and J. A. Jane: Prediction of cavernous sinus invasion in patients with Cushing's disease by magnetic resonance imaging. J. Neurosurg., 130(5), 1593-1598 (2019) doi:10.3171/2018.2.JNS172704

17. R. D. Dickerman and E. H. Oldfield: Basis of persistent and recurrent Cushing disease: an analysis of findings at repeated pituitary surgery. J. Neurosurg., 97(6), 1343-9 (2002) doi:10.3171/jns.2002.97.6.1343

18. R. R. Lonser, A. Ksendzovsky, J. J. Wind, A. O. Vortmeyer and E. H. Oldfield: Prospective evaluation of the characteristics and incidence of adenoma-associated dural invasion in Cushing disease. $J$. Neurosurg., 116(2), 272 (2012)

19. R. Dallapiazza, A. E. Bond, Y. Grober, R. G. Louis, S. C. Payne, E. H. Oldfield and J. J. Jane: Retrospective analysis of a concurrent series of microscopic versus endoscopic transsphenoidal surgeries for Knosp Grades 0-2 nonfunctioning pituitary macroadenomas at a single institution. J. Neurosurg., 121(3), 511-7 (2014) doi:10.3171/2014.6.JNS131321

20. S. Dhandapani, H. Singh, H. M. Negm, S. Cohen, V. K. Anand and T. H. Schwartz: Cavernous Sinus Invasion in Pituitary Adenomas: Systematic Review and Pooled Data Meta-Analysis of Radiologic Criteria and Comparison of Endoscopic and Microscopic Surgery. World Neurosurg., 96, 36-46 (2016) doi:10.1016/j.wneu.2016.08.088

21. S. S. Shin, P. A. Gardner, J. Ng, A. H. Faraji, N. Agarwal, S. Chivukula, J. C. Fernandez-Miranda, C. H. Snyderman and S. M. Challinor: Endoscopic Endonasal Approach for Adrenocorticotropic HormoneSecreting Pituitary Adenomas: Outcomes and Analysis of Remission Rates and Tumor Biochemical Activity with Respect to Tumor Invasiveness. World Neurosurg., 102, 651-658.e1 (2017) doi:10.1016/j.wneu.2015.07.065

22. C. Dai, Y. Fan, X. Liu, X. Bao, Y. Yao, R. Wang and M. Feng: Predictors of Immediate Remission after Surgery in Cushing's Disease Patients: a large retrospective study from a single center. Neuroendocrinology (2020) doi:10.1159/000509221

23. Y. Liu, X. Liu, X. Hong, P. Liu, X. Bao, Y. Yao, B. Xing, Y. Li, Y. Huang, H. Zhu, L. Lu, R. Wang and M. Feng: Prediction of Recurrence after Transsphenoidal Surgery for Cushing's Disease: The Use of Machine Learning Algorithms. Neuroendocrinology, 108(3), 201-210 (2019) doi:10.1159/000496753

24. L. B. Yap, H. E. Turner, C. B. Adams and J. A. Wass: Undetectable postoperative cortisol does not always predict long-term remission in Cushing's disease: a single centre audit. Clin Endocrinol (Oxf), 56(1), 25-31 (2002) doi:10.1046/j.0300-0664.2001.01444.x

25. D. A. Abu, O. N. Singh, N. A. Al, W. H. Farah, P. Barrionuevo, M. Sarigianni, A. B. Mohabbat, K. Benkhadra, L. B. Carranza, M. R. Gionfriddo, Z. Wang, K. Mohammed, A. T. Ahmed, T. A. Elraiyah, Q. 
Haydour, F. Alahdab, L. J. Prokop and M. H. Murad: PREDICTORS OF BIOCHEMICAL REMISSION AND RECURRENCE AFTER SURGICAL AND RADIATION TREATMENTS OF CUSHING DISEASE: A SYSTEMATIC REVIEW AND META-ANALYSIS. Endocr. Pract., 22(4), 466-75 (2016) doi:10.4158/EP15922.RA

26. P. Dolati, D. Eichberg, A. Golby, A. Zamani and E. Laws: Multimodal Navigation in Endoscopic Transsphenoidal Resection of Pituitary Tumors Using Image-Based Vascular and Cranial Nerve Segmentation: A Prospective Validation Study. World Neurosurg., 95, 406-413 (2016) doi:10.1016/j.wneu.2016.06.008

27. T. J. Owen, A. V. Chen, S. Frey, L. G. Martin and T. Kalebaugh: Transsphenoidal surgery: accuracy of an image-guided neuronavigation system to approach the pituitary fossa (sella turcica). Vet. Surg., 47(5), 664-671 (2018) doi:10.1111/vsu.12906

28. O. Solheim, T. Selbekk, L. Lovstakken, G. A. Tangen, O. V. Solberg, T. F. Johansen, J. Cappelen and G. Unsgard: Intrasellar ultrasound in transsphenoidal surgery: a novel technique. Neurosurgery, 66(1), 173-85; discussion 185-6 (2010) doi:10.1227/01.NEU.0000360571.11582.4F

29. B. Zhao, Y. Wei, G. Li, Y. Li, Y. Yao, J. Kang, W. Ma, Y. Yang and R. Wang: Extended transsphenoidal approach for pituitary adenomas invading the anterior cranial base, cavernous sinus, and clivus: a single-center experience with 126 consecutive cases. J. Neurosurg., 112(1), 108 (2010)

30. P. Witek, G. Zielinski, K. Szamotulska, M. Maksymowicz and G. Kaminski: Clinicopathological predictive factors in the early remission of corticotroph pituitary macroadenomas in a tertiary referral centre. Eur. J. Endocrinol., 174(4), 539-49 (2016) doi:10.1530/EJE-15-1226

31. R. M. Starke, D. L. Reames, C. J. Chen, E. R. Laws and J. J. Jane: Endoscopic transsphenoidal surgery for cushing disease: techniques, outcomes, and predictors of remission. Neurosurgery, 72(2), 240-7; discussion 247 (2013) doi:10.1227/NEU.0b013e31827b966a

32. L. J. Blevins, J. H. Christy, M. Khajavi and G. T. Tindall: Outcomes of therapy for Cushing's disease due to adrenocorticotropin-secreting pituitary macroadenomas. J Clin Endocrinol Metab, 83(1), 63-7 (1998) doi:10.1210/jcem.83.1.4525

33. E. Valassi, B. M. K. Biller, B. Swearingen, F. Pecori Giraldi, M. Losa, P. Mortini, D. Hayden, F. Cavagnini and A. Klibanski: Delayed Remission after Transsphenoidal Surgery in Patients with Cushing's Disease. The Journal of Clinical Endocrinology \& Metabolism, 95(2), 601-610 (2010) doi:10.1210/jc.2009-1672

34. P. C. Johnston, L. Kennedy, A. H. Hamrahian, Z. Sandouk, J. Bena, B. Hatipoglu and R. J. Weil: Surgical outcomes in patients with Cushing's disease: the Cleveland clinic experience. Pituitary, 20(4), 430-440 (2017) doi:10.1007/s11102-017-0802-1

35. P. Valderrábano, J. Aller, L. García-Valdecasas, J. García-Uría, L. Martín, N. Palacios and J. Estrada: Results of repeated transsphenoidal surgery in Cushing's disease. Long-term follow-up. Endocrinología y Nutrición, 61(4), 176-183 (2014) doi:10.1016/j.endonu.2013.10.008

36. N. Qiao: Outcome of endoscopic vs microsurgical transsphenoidal resection for Cushing's disease. Endocrine Connections, 7(1), R26-R37 (2018) doi:10.1530/EC-17-0312 
37. L. H. A. Broersen, N. R. Biermasz, W. R. van Furth, F. de Vries, M. J. T. Verstegen, O. M. Dekkers and A. M. Pereira: Endoscopic vs. microscopic transsphenoidal surgery for Cushing's disease: a systematic review and meta-analysis. Pituitary, 21(5), 524-534 (2018) doi:10.1007/s11102-018-0893-3 